\title{
Implementation of Integrated Disease Surveillance and Response (IDSR) in Swaziland
}

\author{
Siphiwe M. Shongwe-Gama*2, Thulani Maphosa², Phinda Khumalo, Vusie Lokotfwako, \\ Nhlanhla Nhlabatsi ${ }^{1}$, Ruben Sahabo ${ }^{2}$ and Harriet Nuwagaba-Biribonwoha ${ }^{2,3}$
}

${ }^{1}$ Epidemiology and Disease Control Unit, Ministry of Health, Mbabane, Swaziland; ${ }^{2}$ ICAP at Columbia University, Swaziland, Mbabane, Swaziland; ${ }^{3}$ Department of Epidemiology, Columbia University, Mailman School of Public Health, Columbia, DC, USA

\section{Objective}

To strengthen public health surveillance and monitor implementation of Integrated Disease Surveillance and Response in the Kingdom of Swaziland.

\section{Introduction}

Swaziland adopted the Integrated Disease Surveillance and Response (IDSR) strategy in 2010 to strengthen Public Health Surveillance (PHS) that fulfills International Health Regulations (2005) and the Global Health Security Agenda (GHSA). This strategy allows the Ministry of Health $(\mathrm{MoH})$, Epidemiology and Disease Control Unit (EDCU) to monitor, prevent and control priority diseases in the country. We used a health systems strengthening approach to pilot an intervention model for IDSR implementation at five hospitals in Swaziland over a pilot phase of three months.

\section{Methods}

Our intervention included cross-country IDSR trainings, sensitizations and onsite trainings targeting national and regional health teams for over 250 health workers. The EDCU developed and disseminated standardized case definitions for health facilities (HFs) to detect, confirm and report priority conditions. Trained health care workers were tasked to cascade knowledge sharing and sensitization about IDSR with their HFs during in-service trainings. The facilities were to use IDSR standard case definition as guidelines for diagnosing and reporting cases; submit monthly reports on all priority conditions to Health Management Information System (HMIS) and intensify reporting through immediate disease notification system (IDNS) for all notifiable conditions. Indicators and monitoring tools for disease surveillance and response as recommended by the technical guidelines for IDSR in the African region were developed. The intervention was evaluated at five purposively selected high-volume referral hospitals (attending to $\geq 1500$ to 15000 outpatient visits per month), which also have maternity services.

Structured questionnaires in the form of a monitoring tool, checklists and observations were used to collect data. Quantitatively, monthly reports submitted by the five facilities to HMIS were reviewed and analyzed for completeness and timeliness. Clinic supervisors were identified from outpatient, inpatient, maternity and laboratory departments as key informants to explore successes and challenges of IDSR implementation. Additionally, IDSR officers visited health facilities and observed the registers and reporting forms used to report IDSR priority conditions and the availability of IDSR guidelines.

\section{Results}

The five HFs submitted monthly reports from June to August 2017 with a calculated completeness of $80 \%$ in June $2017,60 \%$ in July and $40 \%$ in August. Timeliness was calculated was at $20 \%$ in June, $20 \%$ in July and $40 \%$ in August. IDSR officers observed that all five HFs document cases of priority diseases in registers during consultations and use daily tally sheets. However, it was observed that diseases reported through the immediate diseases notification system were not all documented in the morbidity registers and vice versa. Health workers reported to be unaware about all diseases that require immediate notification to trigger investigation, hence some disease like perinatal deaths were never notified through the IDNS system during the period of evaluation. All five hospitals reported not utilizing the standard cases definitions provided to identify and report IDSR priority diseases.

\section{Conclusions}

The proportion of completeness and timeliness from the five HFs during the evaluation period was low compared to WHO recommended standards of $>=80 \%$ from all HFs. This therefore, poses challenges in monitoring and responding to the priority conditions as per IDSR standards and recommendations. All five hospitals reported not utilizing the standard cases definitions to identify and report IDSR priority diseases and this poses challenges in comparison of data across sites, monitoring priority diseases, conditions and events and also identifying the alert or epidemic thresholds. There is need to capacitate more health workers on IDSR for Swaziland to strengthen PHS and be able to prevent and control public health threats timely.

\section{Keywords}

Integrated Disease Surveillance and Response (IDSR); Timeliness and Completeness; Immediate Diseases Notification System (IDNS); Reporting; Health Facilities

\section{Acknowledgments}

This work is supported by the President's Emergency Plan for AIDS Relief (PEPFAR) through the Centers for Disease Control and Prevention under the terms of Cooperative Agreement Number 1U2GGH001271. Its contents are solely the responsibility of the authors and do not necessarily represent the official views of PEPFAR or the Centers for Disease Control and Prevention.

\section{*Siphiwe M. Shongwe-Gama}

E-mail: mabakass@yahoo.com 